\title{
Kocuria kristinae-caused sepsis in an infant with congenital tufting enteropathy
}

\author{
Malik Aydin ${ }^{1,2}$, Rainer Ganschow ${ }^{1}$, Martin Jankofsky ${ }^{1}$ \\ ${ }^{1}$ Department of General Pediatrics, University Children's Hospital, Bonn; ${ }^{2}$ HELIOS University Medical Center Wuppertal, \\ Children's Hospital, Center for Clinical and Translational Research (CCTR), Center for Biomedical Education and Recearch, \\ University of Witten-Herdecke, Germany.E-mail: malik. aydin@uni-wh.de \\ Received: 12th July 2016, Revised: 6th January 2017, Accepted: 1st March 2017
}

\begin{abstract}
SUMMARY: Aydin M, Ganschow R, Jankofsky M. Kocuria kristinae-caused sepsis in an infant with congenital tufting enteropathy. Turk J Pediatr 2017; 59: 93-96.

Congenital tufting enteropathy (CTE) is characterized by the early-onset of chronic diarrhea and the inability to develop. It is a rare congenital disease with a low prevalence of 1:50,000 - 100,000 live births p.a. The histopathology is characterized by villous atrophy and the characteristic epithelial tufts. Recent identification of causative mutations in EpCAM has enhanced our understanding of this disease. Due to its severe clinical course, patients are dependent on parenteral nutrition to thrive successfully. Catheter-associated blood stream infections have become the primary problem for pediatric patients. Infections with Kocuria kristinae are rare. This report is about a 3-month-old girl with CTE suffering from a central venous catheter related mono-sepsis by $K$. kristinae. A sepsis therapy with meropenem and vancomycin improved her general state rapidly. Only few cases in the literature with CTE and $K$. kristinae are described. To the best of our knowledge, this is the first report presenting two coincidences in one case.
\end{abstract}

Key words: congenital tufting enteropathy, congenital intestinal enteropathy, catheter sepsis, Kocuria kristinae, ЕрCAM.

Congenital intestinal epithelial dysplasia, also known as congenital tufting enteropathy (CTE), is a congenital disease with an autosomal recessive inheritance and characterized by intractable watery diarrhea, emesis and failure to thrive; patients suffering from this disorder are mostly dependent on parenteral nutrition ${ }^{1,2}$. The prevalence of CTE is low $(1 / 50,000$ to 100,000 live births p.a.) and seems to be higher in consanguineous Araborigin families ${ }^{3}$. Neither inflammation nor infections causes CTE ${ }^{4}$. The histopathology shows a crypt hyperplasia, villous atrophy, and epithelial tufts in the small intestine; the enterocytes are formed as a teardrop $5,1,2,6,7,8$. Recent identification of causative mutations of genes such as EPCAM and SPINT2 extend our knowledge about this disease ${ }^{9,4}$.

Catheter-associated blood stream infections constitute a significant part of nosocomial infections and are the main complication of treatment ${ }^{10,11}$. Many pathogens may cause catheter-related infections, but gram-positive and coagulase-negative Staphylococcus spp. are mostly responsible for CVC infections $(37.8 \%$ of reported cases), followed by Enterococcus (11.2\%), Staphylococcus aureus (9.3\%), Enterobacter spp. (6.2\%), Candida albicans (5.5\%), Pseudomonas aeruginosa $(4.9 \%)$ and Klebsiella pneumonia $(4.1 \%)^{11}$.

Comparatively, infections caused by opportunistic pathogens are rare ${ }^{11}$. Risk factors for infections due to opportunistic pathogens include young age ( $<2$ months of age), underlying diseases, parenteral nutrition or preexisting infections ${ }^{11}$. Kocuria kristinae is a gram (-) and catalase-positive and coagulasenegative bacterium that belongs to the family of Micrococcacae ${ }^{12,13}$. It is widely distributed and is found commonly on the physiological human skin and oral flora ${ }^{13}$. Cases of Kocuria infections are limited in the literature ${ }^{13}$. Most of those infections have been detected in the past in hospitalized patients with severe underlying diseases or those who had implanted catheters or a suppressed immunity ${ }^{13}$. There are only 
few cases of catheter-associated infections in chronically ill patients caused by Kocuria kristinae and no case report in a patient with CTE $14,15,16$.

This is the first publication with two coincidences of a patient with CTE and a catheter sepsis with Kocuria kristinae.

\section{Case Report}

A seven-day-old girl developed jaundice, sucking weakness, watery diarrhea, acidosis and apathy. She was then brought to the Children's Hospital in Bahrain. Under the suspicion of sepsis and dehydration, she was treated with Ampicillin and Cefotaxime IV for five days. An initial metabolic acidosis was corrected with parenteral fluid therapy. After improving her general state of health, she developed the symptoms listed above once again in course. Due to elevated infectious parameters and an infectious source in central venous catheter (CVC) (positive results in blood cultures for coagulase-negative Staphylococcus spp.), she started to get an antibiotic therapy with vancomycin IV and a parenteral fluid therapy. Under this therapy, her general state of health stabilized. Information on diagnostic tool, and clinical description of the hospital stay in her native country was presented us.

However, at the age of three months, she was then brought to our Children's Hospital with persistent watery diarrhea and physical development problems that required further evaluation.

Our patient was spontaneously born at 36 weeks of gestation with a birth weight of 2500 g. The parents were ethnically Arab (Bahrain), consanguineous and healthy. The parents also had a healthy son. Another son died at the age of 2 months and presented the same symptoms as our patient. Anamnestically, the first cousin to the mother had a total parenteral nutrition-dependent enteropathy with a finally unknown diagnosis.

The current body weight of our patient was $2880 \mathrm{~g}$ (1500 $\mathrm{g}<3^{\text {rd }}$ percentile) and her length was $49 \mathrm{~cm}\left(5 \mathrm{~cm}<3^{\text {rd }}\right.$ percentile $)$. Chemical analysis, screening for metabolic diseases and immunoglobulins showed normal results. Immunological deficiencies could be excluded. Sonographically, the abdomen presented, except of some liquid-filled intestinal loops, normal morphological conditions. Stool tests for pathogens and electrolytes were normal. The swabs (skin, anal and nasal swabs) from the patient showed normal results. Duodenal and colonic biopsies as well as immunehistochemical analysis confirmed our suspected diagnosis of Congenital tufting enteropathy (CTE). A punctuate keratitis, which is common in patients with CTE, was not seen. The diet was managed enterally, via nasogastric tube, and parenterally.

During the preparation for the implantation of the Broviac catheter for total parenteral nutrition, her clinical course became hemodynamically unstable; she developed a sepsis with disseminated intravascular coagulation. Blood cultures from central-venous and peripheral vein catheter for pathogen detection were gathered. Urine samples and cultures were sterile. Under the suspicion of central venous catheter sepsis (CVCS), we decided to remove the "old-existing" catheter, which was more than 10 days old - this was performed by our colleagues in Bahrain (a detailed material analysis/type of material etc. of the central catheter did not exist). We started with a classical broad antibiotic therapy with meropenem and vancomycin IV through the peripheral venous catheter. Within 48 hours after antibiotic therapy, her clinical situation improved rapidly. Blood cultures obtained from CVC and peripheral vein showed a positive detection of the bacterial growth of Kocuria kristinae and S. epidermidis. The pathogen growth in the central blood culture was significantly faster than in the peripheral blood culture (differential time to positivity $>2$ hours), which means that an infection of the CVC is highly probable.

The sensitivity of the tested antibiotics, such as meropenem and vancomycin as well as Teicoplanin, Ciprofloxacin, Linezolid, Gentamicin, Ampicillin, and Penicillin G were sensitive. Considering the critical clinical situation, we decided to continue the broad antibiotic therapy as we did not want to change the antibiotic therapy into a monotherapy till the Broviac catheter (Lifecath ${ }^{\circledR}, 4.2$ F, 1 lumen) was finally implanted laparoscopically into the left internal iliac vein during this period (implantation after antibiotic therapy within 14 days).

A control culture of the blood was not 
performed due to the improved general state of health under the antibiotic regimen. The patient started to get the parenteral nutrition through the Broviac catheter on the same day.

Due to the parenteral nutrition through the Broviac catheter, our patient started to increase in weight and, since then, she is getting the parenteral nutrition through the Broviac catheter.

\section{Discussion}

Central venous catheter sepsis (CVCS) are the primary problem. ${ }^{11}$ There are many studies in the literature of pathogens causing CVCS in adult patients, but there are only few studies about pediatric patients ${ }^{11}$. Many factors such as young age ( $<2$ months), low birth weight, underlying chronic diseases, long hospital stay, peripheral or central catheter, and total parenteral nutrition pose a high risk for catheter sepsis - especially for predisposed opportunistic pathogens $17,18,11$.

Kocuria kristinae, as a facultative pathogen, is to be found in the human oral and skin flora. ${ }^{19}$ CVCS caused by Kocuria kristinae is still limited in the literature ${ }^{19}$. There are only few cases which describe infections caused by that pathogen, e.g. the first publication was a Kocuria kristinae-caused infection in a patient with ovarian carcinoma ${ }^{14,17}$, followed by an immunocompromised patient receiving total parenteral nutrition ${ }^{19}$, or a patient with acute cholecystitis $^{20}$. There is only one study, which summarizes infections by Kocuria kristinae in premature neonates and infants ${ }^{17}$.

Congenital tufting enteropathy (CTE) is a rare disease with an extraordinary clinical history ${ }^{4}$. Opportunistic infections occurred in patients primarily with chronic diseases or an impaired immune system ${ }^{11}$. Catheter-associated infections caused by common pathogens (see also below) are primarily treated empirically ${ }^{21}$. In patients with a chronic disease, such as CTE, opportunistic pathogens, e.g. Kocuria kristinae, must be taken into the therapeutic consideration and quickly given an adequate, effective form of therapy.

The general state of health of our patient improved rapidly under this therapy. Our clinical findings showed us a CVCS. Currently, our patient is almost 12 months old and her clinical situation has improved under the parenteral nutrition very well; she is developing very well, with a few minor complications. Our goal is to help her develop as well as we can, and to stall the small intestine transplantation that was recently discussed as controversial in the literature.

We report a case of CVCS with Kocuria kristinae and S. epidermidis in a three-month-old female infant with CTE. To the best of our knowledge, this is the first reported case of the coincidences of CVCS caused by Kocuria kristinae in a patient with CTE

\section{REFERENCES}

1. Cuenod B, Brousse N, Goulet O, et al. Classification of intractable diarrhea in infancy using clinical and immunohistological criteria. Gastroenterology 1990; 99: 1037-1043.

2. Goulet OJ, Brousse N, Canioni D, Walker-Smith JA, Schmitz J, Phillips AD. Syndrome of intractable diarrhea with persistent villous atrophy in early childhood: a clinicopathological survey of 47 cases. J Pediatr Gastoenterol Nutr 1998; 26: 151-161.

3. Goulet O, Salomon J, Ruemmele F, de Serres NP, Brousse N. Review: Intestinal epithelial dysplasia (tufting enteropathy). Orphanet J Rare Dis 2007; 2: 20.

4. Sivagnanam M, Schaible T, Szigeti R et al. Further evidence for EpCAM as the gene for congenital tufting enteropathy. Am J Med Genet A 2010; 152A: 222-224.

5. Beck NS, Kang IS, Suh YL. Protacted diarrhea: results of the five-year survey in a tertiary hospital in Korea. J Korean Med Sci 2001; 16: 736-741.

6. Ko JS, Seo JK, Shim JO, Hwang SH, Park HS, Kang $\mathrm{GH}$. Tufting enteropathy with EpCAM mutations in two siblings. Gut Liver, 2010; 4: 407-410.

7. Mueller JL, McGeough MD, Pena CA, Sivagnanam M. Functional consequences of EpCAM mutation in mice and men. Am J Physiol Gastrointest Liver Physiol 2014; 306: G278-G288.

8. Sherman PM, Mitchell DJ, Cutz E. Neonatal enteropathies: defining the causes of protacted diarrhea of infancy. J Pediatr Gastroenterol Nutr 2004; 38: 1626.

9. Salomon J, Goulet O, Canioni D et al. Genetic characterization of congenital tufting enteropathy: EpCAM associated phenotype and involvement of SPINT2 in the syndromic form. Hum Genet 2014; 133: 299-310.

10. Bentur HN, Dalzell AM, Riordan FAI. Central venous catheter infection with Bacillus pumilus in an immunocompetent child: a case report. Ann Clin Microbiol Antimicrob 2007; 6: 12.

11. Newman CD, PA-C. Catheter-Related Bloodstream Infections in the Pediatric Intensive Care Unit. Semin Pediatr Infect Dis 2006; 17: 20-24. 
12. Stackebrandt E, Koch C, Gvozdiak O, Schumann P. Taxonomic dissection of the genus Micrococcus: Kocuria gen. nov., Nesterenkonia bgen. Nov. Kytococcus gen. nov. Dermacoccus gen. nov., and Micrococcus Cohn 1872 gen. emend. Int J Syst Bacteriol 1995; 45: 682692.

13. Tewari R, Dudeja M, Das AK, Nandy S. Kocuria kristinae in catheter associated urinary tract infection: A Case Report. J Clin Diagn Res 2013; 7: 1692-1693.

14. Basaglia G, Carretto E, Barbarini D et al. Catheterrelated bacteremia due to Kocuria kristinae in a patient with ovarian cancer. J Clin Microbiol 2002; 40: 311313.

15. Lai CC, Wang JY, Lin SH et al. Catheter-related bacteriaemia and infective endocarditis caused by Kocuria species. Clin Microbiol Infect 2011; 17: 190192.

16. Martinaud C, Gaillard T, Brisou P, Gisserot O, de Jaureguiberry JP. Bacteremia caused by Kocuria kristinae in a patient with acute leukemia. Med Mal Infect 2008; 38: $165-166$.
17. Chen, HM, Chi H, Chiu NC, Huang FY. Kocuria kristinae: A true pathogen in pediatric patients. J Microbiol Immunol Infect 2015; 48: 80-84.

18. Haque KN, Khan MA, Kerry S, Stephenson J, Woods G. Pattern of culture-proven neonatal sepsis in a district general hospital in the United Kingdom. Infect Control Hosp Epidemiol 2004; 25: 759-764.

19. Dunn R, Bares S, David MZ. Central venous catheter-related bacteremia caused by Kocuria kristinae: case report and review of the literature. Ann Clin Microbiol Antimicrob 2011; 10: 31.

20. Ma ES, Wong CL, Lai KT, Chan EC, Yam WC, Chan AC. Kocuria kristinae infection associated with acute cholecystitits. BMC Infect Dis 2005; 5:60.

21. Simon A, Bode U, Beutel K. Diagnosis and treatment of catheter-related infections in paediatric oncology: an update. Clin Microbiol Infect 2006; 12: 606-620. 